\section{Pediatric Inflammatory Multisystem Syndrome Temporally Associated With COVID-19}

We read with interest the recent systematic review written by Meena, et al. [1], wherein the authors have highlighted the clinical features and outcome of the severe acute respiratory syndrome coronavirus 2 (SARS-CoV-2) infection in children. The review covers several aspects related to SARS-CoV-2 infection in children. However, recent emergence of a new clinical syndrome in children in association with SARS-CoV-2 infection also needs a special mention. This pediatric inflammatory multisystem syndrome (PIMS) is characterized by an unusual febrile illness with associated features suggestive of Kawasaki disease, toxic shock syndrome, myocardial dysfunction, or multi-organ failure [2-4]. The diagnostic criteria proposed by the Centre for Disease Control (CDC); World Health Organization (WHO) and Royal College of Paediatrics and Child Health are also not uniform [2-4], signifying a possible geographical difference in the spectrum of clinical manifestations.

A large proportion of children with coronavirus disease 2019 (COVID-19) fall in mild disease category [1]. On the contrary, cases of PIMS-TS reported so far have shown a rather severe course of illness with five deaths out of approximately 300 cases that have been reported [5]. Only up to $70 \%$ of PIMS individuals reported so far were either RTPCR and/or serology positive [6]. This signifies that a positive PCR is not mandatory [3] for diagnosis of PIMS as even contact with a confirmed or suspected case of COVID-19 is enough to make the diagnosis $[2,4]$.

Balasubramanian, et al. [7] recently reported one case of PIMS from India, who was successfully managed with intravenous immunoglobulin (IVIg) and additional immunosuppressants, and also had positive nasopharyngeal RT- PCR.

We wish to highlight that PIMS is a severe spectrum of SARS-CoV-2 infection in children. This syndrome needs early recognition and aggressive management.

Published online: July 15, 2020; PII: S097475591600209

\author{
Murugan Sudhakar ANd ANKur Kumar JindaL* \\ Allergy Immunology Unit, \\ Department of Pediatrics, \\ Advanced Pediatrics Centre, \\ PGIMER, Chandigarh 160 012, India. \\ *ankurjindal11@gmail.com
}

\section{REFERENCES}

1. Meena J, Yadav J, Saini L, Yadav A, Kumar J. Clinical features and outcome of SARS-CoV-2 infection in children: A systematic review and meta-analysis. Indian Pediatr. 2020; S097475591600203 [E-pub ahead of print].

2. Multisystem Inflammatory Syndrome (MIS-C). Information for Healthcare Providers about Multisystem Inflammatory Syndrome in Children. Case Report Form. 2020;2-4. Available from: https://emergency.cdc.gov/han/ 2020/han00432.asp. Accessed July 6, 2020.

3. Royal College of Paediatrics and Child Health Guidance: Paediatric Multisystem Inflammatory Syndrome Temporally Associated with COVID- 19. Available from: https://www.rcpch.ac.uk/sites/default/files/2020-05/ COVID-19-Paediatric-multisystem-\%20inflammatory\% 20syndrome-20200501.pdf. Accessed July 6, 2020.

4. World Health Organization. Multisystem inflammatory syndrome in children and adolescents with COVID-19: Scientific brief, 15 May 2020. Available from: https:// apps.who.int/iris/handle/10665/332095. Accessed July 6, 2020.

5. European Centre for Disease Prevention and Control Country Experts. Rapid Risk Assessment: Paediatric inflammatory multisystem syndrome and SARS-CoV-2 infection in children. Available from: https:// www.ecdc.europa.eu/sites/default/files/documents/covid19-risk-assessment. Accessed July 6, 2020.

6. Feldstein LR, Rose EB, Horwitz SM, et al. Multisystem inflammatory syndrome in US children and adolescents. $\mathrm{N}$ Engl J Med. 2020;10.1056/NEJMoa2021680 [published online ahead of print].

8. Balasubramanian S, Nagendran TM, Ramachandran B, Ramanan AV. Hyper-inflammatory syndrome in a child with covid-19 treated successfully with intravenous immunoglobulin and tocilizumab. Indian Pediatr. 2020;S097475591600180 [E-pub ahead of print]. 